\title{
Los canales infantiles politemáticos de televisión digital en España: análisis y clasificación
}

Polithematic Children's Channels of Digital Television in Spain: Analysis and Classification

Irene Melgarejo. Universidad de Murcia

María del Mar Rodríguez. Universidad de Murcia

Recibido: 13-XII-2010 - Aceptado: 7-III-2011

Resumen:

Los nuevos aportes tecnológicos y el desarrollo de la digitalización han hecho posible que hoy día exista una "amplia" gama de Canales Infantiles Politemáticos de televisión que se han convertido en una alternativa para los más pequeños. Con esta investigación, donde se estudian parámetros como el género y el formato, la publicidad y la autopromoción o la segmentación de edades y el curriculum escolar se muestra el panorama existente en España en cuanto a canales infantiles; para ello se ha diseñado una metodología de estudio basada en la triangulación de métodos científicos donde convergen diversas técnicas, lo que nos permite obtener una aproximación a la realidad de los Canales Infantiles Politemáticos.

Palabras clave:

Canal, definición, infancia, televisión, Politemático

Abstract:

New technologies advances and the development of the digitalization have made possible that today a large range of polithematic children's channels, that have become an alternative for infants, exists. This research, which studies parameters such as genre and format, advertising and self-promotion and age segmentation and school curriculum, shows the Spanish outlook about children's channels. For doing that, we have been designing a study methodology based in the triangulation of scientific methods, where several techniques that allows to get an approximation to the polithematic children's channels reality converges.

Keywords:

Channel, definition, childhood, television, Polithematic 


\section{Introducción}

Desde la más tierna infancia la televisión nos seduce, nos embriaga y se convierte en una aliada fiel para nuestro tiempo de ocio y relax; tan importante es este medio de comunicación en nuestras vidas que hoy día algunos teóricos se atreven a catalogarla como un elemento más de socialización entre los más pequeños. Lo cierto es que la televisión se ha transformado en poco tiempo en el medio más globalizado, forma parte de los hogares y además el desarrollo de la televisión digital está impulsando la multiplicación de canales temáticos, sobre todo aquellos destinados a un público infantil; un aumento de canales que se hace presente en las plataformas de cable y satélite, que emiten horas interminables de programación, -en ocasiones hasta 24 horas ininterrumpidas-, y donde se aglutinan multitud de contenidos, más para el divertimento que para la formación o la educación de los menores.

Los contenidos programáticos para los menores siempre han formado parte de la historia de la televisión desde su comienzo, sin embargo la lucha encarnizada por conseguir altas cuotas de audiencia entre canales generalistas en nuestro país, los han hecho casi desaparecer por completo de las parrillas de programación quedando así un amplio segmento de audiencia desatendido. No obstante, los nuevos aportes tecnológicos y el desarrollo de la digitalización han hecho posible que hoy día exista una "amplia" gama de canales Infantiles Politemáticos que se han convertido en una alternativa para los más pequeños, donde poder encontrar una programación destinada en exclusiva para este tipo de target y adaptada a los gustos, preferencias y necesidades de los niños.

\section{Experiencia de la investigación}

El presente artículo muestra los datos globales derivados de la investigación titulada Canal Infantil Politemático de Televisión Digital: clasificación y aproximación a una definición en la que se estudiaron diversos aspectos relacionados con los canales infantiles de televisión, los contenidos programáticos y la infancia. En ella se recoge una nueva propuesta de definición y terminología más acorde con la realidad de este tipo de canales y donde los hasta ahora llamados canales temáticos infantiles pasarían a denominarse Canales Infantiles Politemáticos ${ }^{1}$.

Existe cierta confusión a la hora de referirnos a los canales infantiles como temáticos; consideramos que se hace un mal uso del término, puesto que no son temáticos en sí, ya que no atienden a una temática determinada sino que las temáticas son diversas pese a que siempre aluden a temas propios de la infancia. Por ello, acuñamos el término Politemático por ajustarse más a la realidad de los canales infantiles de televisión digital, puesto que en ellos se tiende más a la variabilidad y pluralidad de temáticas empleadas en sus contenidos. 
Los canales temáticos en su conjunto vienen siendo estudiados por diversos autores -Mariano Cebrián, Enrique Bustamante, Gema Alcolea, Manuel Palacio, entre otros-. Sin embargo, las aproximaciones que se han hecho sobre los canales temáticos infantiles de televisión siempre han sido un tanto superfluas, por lo que al plantear la investigación de la que forman parte los resultados que se exponen en este artículo, pretendemos adentrarnos en el estudio de los canales infantiles de televisión digital para ofrecer una visión panorámica del espectro de canales que operan en España. Así, la revisión de las investigaciones que nos anteceden, el estudio de las parrillas de programación de este tipo de canales y sus contenidos, entre otros aspectos, nos ayudan en la difícil tarea de desarrollar diversas clasificaciones atendiendo tanto al segmento de edad, al género y al formato, como a los aspectos relacionados con la continuidad y la adaptación de los contenidos al Curriculum Escolar del menor, lo que nos permite ofrecer una aproximación acertada a la definición de Canal Infantil Politemático; una novedosa terminología que acuñamos en nuestro estudio, que representa de forma clara a este tipo de canales y que se ajusta más a la realidad de los mismos.

Por primera vez con esta investigación se propone un estudio analítico, así como una reflexión profunda sobre la totalidad de Canales Infantiles Politemáticos que conforman el espectro televisivo infantil en el ámbito de las plataformas nacionales de televisión digital de pago. Esto supone ampliar los conocimientos sobre el funcionamiento y la estructura de este tipo de canales, así como ofrecer una visión actual y global para seguir avanzando en los estudios sobre programación y contenidos destinados a los menores, ya que se traza un mapa tanto de los canales existentes como de los contenidos abordados por los mismos, de tal modo que podamos contribuir con nuestra investigación a conocer más en profundidad el espectro televisivo español. Por otro lado, los nuevos aportes que presentamos, -tanto en lo que se refiere a los aspectos terminológicos como de la definición de Canal Infantil Politemático de Televisión Digital-, son más acordes con la realidad de los canales que operan hoy día en España. Por lo que contribuciones como las que presentamos en este artículo son útiles para el entorno de la producción y la creación audiovisual destinada para la infancia, al igual que será importante para la comunidad científica y docente, puesto que vienen siendo poco estudiados en el ámbito académico -aunque sí es cierto que cada vez más se está generando un interés colectivo por los contenidos que visionan los más pequeños-. En definitiva, consideramos de vital importancia investigaciones como la que presentamos por ofrecer una visión global y exhaustiva de la totalidad de canales, así como de sus contenidos, géneros, formatos, continuidad, etc., que nos permitirá seguir avanzando en la creación de una televisión adecuada que satisfaga las necesidades de los niños en un futuro próximo. 


\subsection{Objeto de estudio y objetivos}

La exposición de los resultados globales de la investigación ofrece una visión exhaustiva y desde distintos puntos de vista de los principales Canales Infantiles Politemáticos que operan en España. De ahí que nuestro objeto de estudio esté conformado por 11 canales de televisión digital cuya emisión de contenidos va destinada a los menores: Disney Channel, Playhouse Disney, Disney Cinemagic, Disney XD, Cartoon Network, Boomerang, Nickelodeon, Nick jr., Kidsco, Clan TVE y BabyTV². Ya que consideramos que el canal temático como estructura televisiva tiene más entidad de estudio que un simple programa de televisión, lo que nos permite conocer su estructura, funcionamiento, contenidos, públicos, entre muchos otros aspectos, de forma global y por categorías de análisis. Así, la elección de estos 11 canales obedece a una razón práctica: son los principales canales infantiles de televisión digital ofertados por las distintas plataformas que operan en España, ya sean de cable, satélite o ADSL. De este modo, la mayoría de canales recogidos en nuestro objeto de estudio forman parte de las distintas plataformas de televisión activas en nuestro país. No obstante, hemos tomado como referencia a la plataforma por cable de ONO como vía de acceso a estos canales y para poder conocerlos más en profundidad. Por lo tanto, el objeto de estudio queda así delimitado y es lo suficientemente amplio como para tener una visión global del entorno de los canales infantiles de televisión en nuestro país.

Como a través de nuestro estudio pretendemos conocer más de cerca la variedad programática que nos ofrece este tipo de canales infantiles de televisión digital, nos marcamos cuatro objetivos fundamentales que giran en torno a la televisión temática, sus contenidos programáticos y la relación con la infancia:

- $\quad$ Asumiendo la expresión "canal temático infantil”, formular una nueva definición de este tipo de canales, proponiendo una nueva terminología, la de Canal Infantil Politemático como más adecuada para referirnos a ellos.

- $\quad$ Teniendo en cuenta las características, aspectos y contenidos que ha de tener un canal de televisión infantil para poder referirnos con propiedad a esta modalidad de televisión, formular una clasificación de canales infantiles politemáticos.

- Identificar los distintos segmentos de edad del público objetivo al que va destinada la programación de los canales a estudio, puesto que la elección de uno u otro contenido por parte de los programadores de televisión siempre corresponde a las características y a las demandas de su espectador potencial.

Hay que tener en cuenta que desde la finalización del estudio que nos ocupa han aparecido en el panorama audiovisual español dos nuevos Canales Infantiles Politemáticos: Boing y Canal Panda, que sin embargo no han formado parte de esta investigación. 
Conocer la realidad televisiva de este tipo de canales infantiles politemáticos que operan en España a través de la plataforma ONO.

\subsection{Metodología, marco temporal y fuentes}

Hasta el momento la mayoría de estudios relacionados con la televisión y la infancia tienen un carácter puramente cuantitativo; datos numéricos que nos aportan una visión sobre la relación de los menores con la reina de la comunicación, sus gustos, preferencias y hasta los usos. Tomando como referencia esos estudios precedentes, así como todos aquellos que se han elaborado en torno a los canales temáticos, la televisión y la infancia, desarrollamos una investigación basada en la triangulación de métodos científicos para poder obtener resultados tanto cuantitativos como cualitativos con el fin de conseguir un espectro más amplio y global de resultados. De esta forma, se plantea una investigación con carácter descriptivo donde las herramientas metodológicas empleadas son la observación descriptiva como un primer punto de partida para conocer cómo se manifiesta este medio de comunicación. Las entrevistas en profundidad a los directivos de los respectivos canales a estudio para conocer de primera mano los requisitos y aspectos tenidos en cuenta por las empresas a la hora de programar los contenidos para los menores y confeccionar el canal, lo que nos ayuda a una posible definición de Canal Infantil Politemático. Y el análisis de contenidos de todos y cada uno de los productos audiovisuales que ofertan este tipo de canales, que nos han ayudado a estudiar los géneros y los formatos, los segmentos de edad y el Curriculum Escolar, así como la continuidad y autopromoción de los mismos. De esta forma, para el análisis de contenido, y tomando como referencia a diversos autores relevantes en cada una de las materias analizadas, se han confeccionado una serie de tablas que atienden a esas características: para el análisis referente al género y al formato hemos tenido en cuenta los aportes de Barroso (1996) y Sánchez (2002), para el análisis de la continuidad a González (2005), López (2005), Galindo (2004), etc., y por último para la segmentación de edades y el Currículum Escolar hemos tomado como referencia los estudios de Tomás De Andrés (2006) y Verónica Marín (2005).

Como ya hemos anunciado en líneas anteriores la investigación Canal Infantil Politemático de Televisión Digital: clasificación y aproximación a una definición trata de ser un reflejo descriptivo tanto de los canales infantiles politemáticos de televisión digital existentes, como de los contenidos que conforman los mismos. De ahí que nuestro estudio quede acotado de la siguiente forma: en primer lugar, se ha tomado como referencia la plataforma por cable de ONO por aglutinar en sus diferentes ofertas de paquetes temáticos el mayor número de canales infantiles. De esta forma, consideramos que el espectro de canales que nos ofrece la plataforma ONO es lo suficientemente amplio como para obtener una visión lo más aproximada a la re- 
alidad televisiva infantil en España, ya que nos ofrece 11 canales-CLAN TVE, Disney Channel, Playhouse Disney, Disney Cinemagic, Disney XD, Cartoon Network, Boomerang, Nickelodeon, Nick Jr., Kidsco, BabyTV-.Y en segundo lugar, para desarrollar la investigación se han analizado 7 días de la parrilla de programación de cada uno de los canales a estudio, en total 77 días de contenidos que nos aportarán una visión de la estructura programática de los respectivos canales. Por tanto, se han tomado como muestra 54 horas de programación de cada uno de estos canales para desarrollar un análisis minucioso de los contenidos abordados en los 11 canales a estudio. Así, se analizan en total 594 horas de productos audiovisuales con el fin de poder desarrollar diversas clasificaciones que atiendan a los parámetros de género, formato, segmentación, continuidad, currículum escolar, entre otros aspectos. En este sentido, las muestras recogidas para el consiguiente análisis (tanto el que se refiere al contenido como al estudio descriptivo de las parrillas de programación) abarcan un marco temporal amplio que va desde el 5 de mayo hasta el 25 julio de 2010. Además, durante ese espacio temporal se han desarrollado también las distintas entrevistas en profundidad a los directivos de dichos canales.

Finalmente, para llevar a buen puerto esta investigación, ha sido necesaria la consulta y revisión de una gran diversidad de autores relevantes en la materia, pues ante todo se pretendía que la investigación fuese lo más completa posible en el tratamiento de las fuentes. De esta forma nuestras referencias son amplias, puesto que van desde aquellos autores más vinculados con el estudio de los modelos y estructuras televisivas como son: Cebrián (2004), Bustamante (1999), Barroso (1996), entre muchos otros, hasta aquellos autores que se centran en el estudio de la infancia, unos más a nivel psicológico como puede ser Piaget (2001) o De Andrés (2006) con sus estudios sobre la Inteligencia Fílmica y otros más prácticos y relacionados con la televisión, como son Tur (2004) o Núñez (2007), entre otros. No obstante, ya que los documentos bibliográficos de los que se dispone hoy día en materia de televisión e infancia son abundantes, se ha tenido que realizar una criba bastante selectiva de autores que nos han servido para confeccionar tanto la parte teórica, como la metodológica y la parte práctica del estudio.

Hemos querido exponer en este epígrafe, grosso modo, cómo se ha desarrollado la investigación en su conjunto para que puedan entenderse los datos reflejados en los siguientes apartados y que declinarán en las pertinentes conclusiones.

\section{Resultados}

Una vez justificada nuestra investigación y habiendo expuesto todos aquellos aspectos relevantes relacionados con el objeto de estudio, los objetivos y la metodología, se exponen los resultados más relevantes 
obtenidos y que constituyen una muestra de lo que son hoy día los Canales Infantiles Politemáticos de Televisión Digital. De esta forma, se atiende a las variables de género y formato, publicidad y autopromoción, y segmentación de edades y Currículum Escolar en cada uno de los canales infantiles politemáticos a estudio (CLAN, Disney Channel, Plahyhouse Disney, Disney Cinemagic, Disney XD, Nickelodeon, Nick Jr., Cartoon Network, Boomerang, Kidsco y Baby TV).

\subsection{Género y formato}

\section{CLAN TVE}

CLAN es un canal infantil que emite 24 horas ininterrumpidas de programación; a lo largo de 7 días encontramos la inclusión en su parrilla de programación de 30 productos audiovisuales diferentes; contenidos que nos permiten encasillarlo como Canal Infantil Politemático y juvenil de entretenimiento ya que el 100\% de sus series, ya sean de dibujos animados (63’33\%) o series de ficción (20\%), atienden a este parámetro. No obstante, es de destacar el alto porcentaje de productos que tiene en cuenta al género educativo para el público más pequeño con un 26’66\% y el de ficción con un 23’33\%.

\section{The Walt Disney Company: Disney Channel, Playhouse Disney, Disney Cinemagic y Disney XD}

Los canales infantiles politemáticos de la compañía Disney tienden al entretenimiento en su gran mayoría; sin embargo encontramos cómo este parámetro se complementa con el de género educativo sobre todo en aquellos canales que se dirigen a un público más pequeño. En Disney Channel encontramos la inclusión en su parrilla de programación de 45 contenidos que pertenecen a los géneros puramente de la ficción (44’44\%), educativos (15’55\%) y musicales (4’44\%). En cuanto a los formatos más utilizados en sus contenidos audiovisuales destacan los dibujos animados con un 48’88\% y las series de ficción que alcanzan el $37 ’ 77 \%$ de la programación de este canal.

Playhouse Disney a diferencia del resto de sus canales hermanos, emite sólo 18 horas diarias de programación. A lo largo de una semana encontramos en su parrilla 47 contenidos diferentes destinados a un público infantil que va desde los 3 a los 6 años de edad. Es ante todo un canal politemático de entretenimiento educativo donde el 55’31 \% de sus series atienden al género educativo que se complementa con el de entretenimiento, lo que nos demuestra que éste no está reñido con la educación. Al ir destinado a un público tan pequeño, el 85’10\% del formato empleado en sus contenidos es el de los dibujos animados frente a un 12 ' $76 \%$ en donde encontramos series protagonizadas por personajes de gomaespuma. 
Disney Cinemagic se caracteriza por ser un canal politemático de ámbito más familiar que tiene siempre como base el género de entretenimiento, y el de ficción con un 44'73\%. Está conformado por 38 contenidos audiovisuales diferentes entre largometrajes (36’84\%) y series de dibujos animados (55'26\%), nacidas a partir de los míticos largometrajes de la casa Disney y que alargan en el tiempo las aventuras de los conocidos personajes de esta compañía creados en un principio para la gran pantalla y que ahora son explotados en televisión.

La parrilla de programación del canal politemático Disney XD se compone de 28 series diferentes a lo largo de una semana; sin alejarse del entretenimiento, sus contenidos se basan en un 28'57\% en la ficción, de ahí que el formato más utilizado sea el de la serie de dibujos animados con un 67 ' $85 \%$ en relación a las series de ficción (21’42\%) y a los largometrajes (7’14\%). Los contenidos en su gran mayoría atienden a series sacadas del mundo del cómic y sus temas hacen especial hincapié en el mundo de la magia, las fuerzas sobrenaturales, etc.

Podemos concluir este apartado destacando que The Walt Disney Company dispone de un Canal Infantil Politemático y juvenil de entretenimiento (Disney Channel), un Canal Infantil Politemático educativo (Playhouse Disney), un canal politemático familiar basado en la ficción de dibujos animados (Disney Cinemagic) y un Canal Infantil Politemático y juvenil de entretenimiento más enfocado a niños que a niñas (Disney XD).

\section{Viacom Inc.: Nickelodeon y Nick JR}

La compañía Viacom Inc. dispone de dos canales infantiles politemáticos o/y juveniles; el primero de ellos, de más larga andadura en el panorama de la televisión temática es Nickelodeon cuya parrilla de programación está compuesta por 23 contenidos distintos y caracterizada por el género del entretenimiento en su totalidad, dejando a un lado los contenidos educativos (4'34\%). De esta forma sólo podemos encontrar dos tipos de formato: dibujos animados (69’56\%) y series de ficción (30’43\%) destinadas a un target más juvenil.

La variedad programática del Canal Infantil Politemático Nick Jr. es muy escasa, ya que a lo largo de 7 días de programación sólo encontramos 7 series infantiles diferentes y de nuevo vemos cómo 5 de estos contenidos atienden al género educativo (71'42\%) junto al del entretenimiento que se muestra clave en todo Canal Infantil Politemático que se precie. De modo que las 7 series infantiles que conforman la parrilla de programación de Nick Jr. se basan en el formato de dibujos animados.

En definitiva encontramos cómo la compañía Viacom Inc. abre su mercado televisivo en nuestro país a través del Canal Infantil Politemático y juvenil de entretenimiento Nickelodeon, más generalista que Nick Jr. 
cuyo segmento viene a ser más reducido y con unas características propias destinadas a la educación de los más pequeños, por tanto hablamos de Nick Jr. como un Canal Infantil Politemático educativo.

\section{Time Warner Company: Cartoon Network y Boomerang}

La apuesta por los géneros de entretenimiento es lo que caracteriza a los canales infantiles politemáticos Cartoon Network y Boomerang, tanto que el 100\% de sus productos audiovisuales atienden a este parámetro. Sin embargo, lo que los diferencia es el segmento de edad del público al que se dirigen y de esta forma Boomerang aboga por un target más infantil (de 3 a 6 años y familiar), mientras Cartoon Network está más enfocado a un público varón de entre 7 a 12 años de edad, de ahí que la inclusión de largometrajes para un público más juvenil sea más acusada (14'28\%) que en el caso de Boomerang donde la ficción ocupa un 9’09\% de la parrilla de programación.

El canal politemático Boomerang está compuesto de 11 series diferentes, de las que el 90'90\% son dibujos animados, mientras en el caso del canal politemático Cartoon Network los contenidos que incluyen en su parrilla son casi el doble-21 productos audiovisuales diferentes durante 7 días de programación-entre los que encontramos sobre todo formatos de dibujos animados (85’71\%).

\section{Kidsco}

El canal politemático Kidsco es ante todo un canal destinado tanto a un público infantil como juvenil, de ahí que podamos encontrar contenidos del género de la ficción (37’03\%), aunque no obstante, la totalidad de sus contenidos (27 productos audiovisuales) son de entretenimiento y sólo un 3'70\% atiende al género musical. Predominan las series de dibujos animados con un 55'55\%, los largometrajes que alcanzan un valor del 25’92\% y las series de ficción con un 14’81\%.

\section{BabyTV}

BabyTV es un canal politemático atípico, de los que no suelen predominar en las ofertas televisivas por incluir en su parrilla de programación contenidos destinados a un público menor de 2 años. Tiene una gran variedad de contenidos ya que su estructura programática está compuesta de 48 productos distintos. Este canal emite las 24 horas al día, adecuando sus productos a cada franja horaria en base a las necesidades de los bebés -se constata la existencia de productos exclusivos para la franja matinal y otros más relajados y musicales para la de noche-.

Así, encontramos cómo sus productos, además de ser de entretenimiento, muestran una clara inclinación hacia el género educativo (39’58\%) y al musical (16’66\%), géneros que son puestos en antena bajo el formato 
del dibujo animado en un 91'66\% de las ocasiones frente a un 8'33\% que atienden a otra serie de formatos -no es de extrañar si tenemos en cuenta que hablamos de niños muy pequeños que gustan de formas sencillas y colores que llamen su atención-.

Tabla I. Resumen Variable Género (en \%)

\begin{tabular}{|c|c|c|c|c|c|c|c|c|c|c|c|c|}
\hline \multirow[b]{2}{*}{ CANALES TV } & \multicolumn{12}{|c|}{ GÉNEROS } \\
\hline & 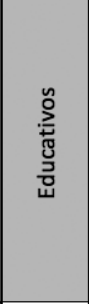 & 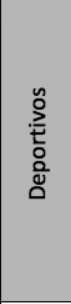 & $\begin{array}{l}\text { y } \\
\frac{\pi}{0} \\
\frac{\pi}{0} \\
\frac{d}{\frac{1}{\pi}} \\
>\end{array}$ & 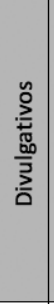 & 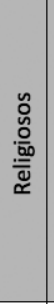 & 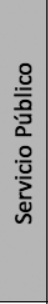 & 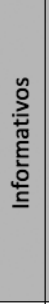 & 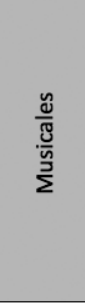 & $\begin{array}{l}\text { 듬 } \\
\text { 은 }\end{array}$ & 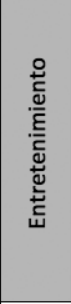 & 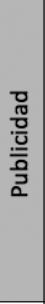 & 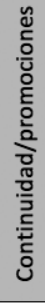 \\
\hline CLAN TVE & 26,66 & 0 & 0 & 0 & 0 & 0 & 0 & 0 & 23,33 & 100 & 0 & 0 \\
\hline DISNEY CHANNEL & 15,55 & 0 & 0 & 0 & 0 & 0 & 0 & 4,44 & 44,44 & 100 & 0 & 0 \\
\hline $\begin{array}{l}\text { PLAYHOUSE } \\
\text { DISNEY }\end{array}$ & 55,31 & 0 & 0 & 0 & 0 & 0 & 0 & 2,12 & 2,12 & 100 & 0 & 0 \\
\hline $\begin{array}{l}\text { DISNEY } \\
\text { CINEMAGIC }\end{array}$ & 0 & 0 & 0 & 0 & 0 & 0 & 0 & 0 & 44,73 & 100 & 0 & 0 \\
\hline DISNEY XD & 0 & 3,57 & 0 & 0 & 0 & 0 & 0 & 0 & 38,57 & 100 & 0 & 0 \\
\hline NICKELODEON & 4,34 & 0 & 0 & 0 & 0 & 0 & 0 & 4,44 & 30,43 & 100 & 0 & 0 \\
\hline NICK JR & 71,42 & 0 & 0 & 0 & 0 & 0 & 0 & 2,12 & 0 & 100 & 0 & 0 \\
\hline $\begin{array}{l}\text { CARTOON } \\
\text { NETWORK } \\
\end{array}$ & 0 & 0 & 0 & 0 & 0 & 0 & 0 & 0 & 14,28 & 100 & 0 & 0 \\
\hline BOOMERANG & 9,09 & 0 & 0 & 0 & 0 & 0 & 0 & 0 & 9,09 & 100 & 0 & 0 \\
\hline KIDSCO & 0 & 0 & 0 & 0 & 0 & 0 & 0 & 3,7 & 37,03 & 100 & 0 & 0 \\
\hline BABY TV & 39,58 & 0 & 0 & 0 & 0 & 0 & 0 & 16,66 & 0 & 95,8 & 0 & 0 \\
\hline
\end{tabular}

(Fuente: elaboración propia) 
Tabla II. Resumen Variable Formato (en \%)

\begin{tabular}{|c|c|c|c|c|c|c|c|}
\hline \multirow[b]{2}{*}{ CANALES TV } & \multicolumn{7}{|c|}{ FORMATOS } \\
\hline & 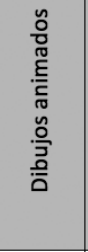 & 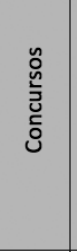 & 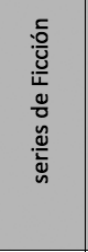 & 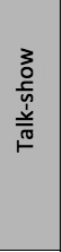 & 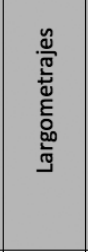 & 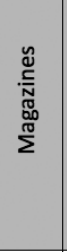 & 气ั๋ \\
\hline CLAN TVE & 63,33 & 0 & 20 & 0 & 3,33 & 0 & 13,33 \\
\hline DISNEY CHANNEL & 48,88 & 2,22 & 37,77 & 0 & 6,66 & 2,22 & 2,22 \\
\hline PLAYHOUSE DISNEY & 85,1 & 0 & 0 & 0 & 2,12 & 0 & 12,76 \\
\hline DISNEY CINEMAGIC & 55,26 & 0 & 7,89 & 0 & 36,84 & 0 & 0 \\
\hline DISNEY XD & 67,85 & 3,57 & 21,42 & 0 & 7,14 & 0 & 0 \\
\hline NICKELODEON & 69,56 & 0 & 30,43 & 0 & 0 & 0 & 0 \\
\hline NICK JR & 100 & 0 & 0 & 0 & 0 & 0 & 0 \\
\hline CARTOON NETWORK & 85,71 & 0 & 0 & 0 & 14,28 & 0 & 0 \\
\hline BOOMERANG & 90,09 & 0 & 0 & 0 & 9,09 & 0 & 0 \\
\hline KIDSCO & 55,55 & 0 & 14,81 & 0 & 25,92 & 0 & 3,7 \\
\hline BABY TV & 91,66 & 0 & 0 & 0 & 0 & 0 & 8,33 \\
\hline
\end{tabular}

(Fuente: elaboración propia)

\subsection{Publicidad y autopromoción}

Con lo que respecta a la ausencia o presencia de estas dos variables -publicidad y autopromoción- podemos destacar que de los 11 canales infantiles politemáticos analizados tan sólo en 4 de ellos -CLAN, Nick Jr., Kidsco y BabyTV- (36’36\%) encontramos la no inclusión de publicidad externa, mientras que un 63'63\% de los canales incluyen en los bloques para la pausa publicitaria spots de productos no relacionados con el propio canal. No obstante, en los 11 canales a estudio (100\% de los casos) nos encontramos la inclusión en sus paquetes de continuidad programática el recurso de la autopromoción del canal politemático, así como de los productos que conforman las parrillas de programación de dichos canales.

Atendiendo más en profundidad a cada uno de los canales infantiles politemáticos, es necesario aclarar que CLAN TVE carece de publicidad externa al tratarse de un canal de ámbito público que se financia con capital del Estado; pese a ello, cuida mucho su imagen a través de la continuidad y por medio de la inclusión de autopromociones de sus series más importantes que refuerzan su imagen de marca, al igual que 
ocurre con los canales Kidsco, Nick Jr. y BabyTv. La única diferencia con respecto a éstos es que son de ámbito privado y pese a ello no incluyen publicidad como forma de financiación; es más, si nos fijamos, la mayoría de canales que no contemplan los spots televisivos dentro de su parrilla se corresponden con aquellos que van destinados a un público preescolar o incluso menor. No ocurre lo mismo con Playhouse Disney, que incluye publicidad pero sólo de productos puramente de la marca Disney enmarcados en pantalla bajo el rótulo de "publicidad", como forma de diferenciar los contenidos audiovisuales de los productos comerciales, o Boomerang que incluye todo tipo de publicidad destinada y pensada para el telespectador infantil.

Es de vital importancia destacar la valoración positiva que los directivos hacen de las pausas publicitarias, no sólo como forma de rentabilizar el canal sino como un elemento más de información para el telespectador:

"No es un elemento que les moleste, según hemos constatado en los focus group que hemos hecho. Para ellos es un elemento de actualidad. Normalmente son anuncios relacionados con su actualidad y con su vida, como puede ser un juguete, unos cromos y demás, con lo cuál no les molesta. Desde luego que nosotros no tenemos pausas publicitarias de 20 minutos. Entonces ellos lo soportan mientras sea reducido, porque intentamos no ser plataformas saturadas de publicidad" (Vincent Sourdeau, Director de canales temáticos Cartoon Network y Boomerang).

"Desde el punto de vista de los anunciantes, realmente se facilita el acceso al público objetivo que van buscando en sus campañas de comunicación. (...) Desde el punto de vista de la audiencia, el público infantil, la publicidad les resulta divertida, no les provoca rechazo y tenemos comprobado que apenas sufrimos fugas representativas de nuestra audiencia cuando entramos en los cortes publicitarios. (...) no sólo se incluye publicidad convencional sino que aportamos una serie de contenidos, de corta duración, que son muy atractivos para la audiencia, como pueden ser los video clips musicales de los principales artistas del canal, que mantienen la atención de la audiencia en todo momento. En ningún caso la saturación publicitaria llega a los niveles de los canales generalistas". (Inés Ramos, Directora los canales Disney).

\subsection{Segmentación de edades y currículum escolar}

\section{CLAN TVE}

CLAN es un Canal Infantil Politemático que tiende hacia lo juvenil y familiar en su franja de tarde/noche, por dirigirse a un segmento bastante amplio de audiencia; tanto es así que el $50 \%$ de sus contenidos van dirigidos a un público de entre 3 a 6 años, mientras el otro 50\% está integrado por productos audiovisuales pensados para un target que va desde los 7 años en adelante. 
Así, el 26’66\% de la programación analizada se corresponde con productos adaptados al Currículum Escolar del menor, en su totalidad correspondientes al Currículum de Infantil, de los que el 75\% atienden a la educación del Conocimiento de sí mismo y autonomía personal, el 87’5\% a Conocimiento del entorno y el 62’5\% a Lenguajes: comunicación y representación . Pese a ello, abundan los programas cuyas temáticas o componentes no están adaptados a ningún tipo de currículum (73’33), que en su mayoría se corresponden con aquellos que van destinados a un público de mayor edad.

\section{The Walt Disney Company: Disney Channel, Playhouse Disney, Disney Cinemagic y Disney XD}

La compañía Disney dispone de un canal más generalista con un segmento más amplio de edades como es Disney Channel que dispone de un 71'11\% de contenidos destinados a un target mayor de 7 años y un 28’88\% de sus productos están pensados para un público más infantil (de 3 a 6 años de edad). De ahí que entre sus contenidos se encuentren aquellos adaptados al Currículum Escolar (15’55\%) y otros que atienden más a la diversión y el entretenimiento y que no están adaptados al mismo (84’44\%).

Playhouse Disney es el canal politemático por excelencia de la compañía Disney pensado en exclusiva para un público en edad preescolar (de 3 a 6 años) y más de la mitad de sus contenidos (55’31\%) atienden a productos audiovisuales adaptados al Currículum Escolar de Infantil, mientras el 44'68\% no atienden al mismo, lo que muestra un claro equilibrio entre contenidos destinados a la diversión y aquellos que introducen elementos educativos.

Figura 1. Canales Disney

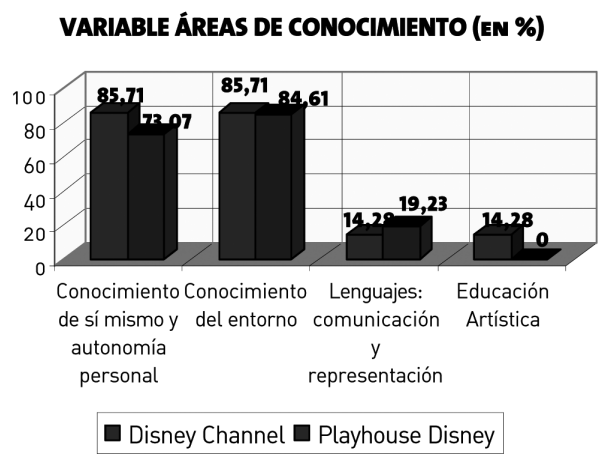

La suma de la mayoría de los porcentajes que se exponen en este apartado supera el 100\% debido a que en ocasiones los ítems de análisis tenidos en cuenta se solapan; en este caso, se observa que los contenidos de una misma serie de televisión atienden tanto a la educación del Conocimiento de sí mismo y autonomía personal, como al Conocimiento del entorno y al de Lenguajes: comunicación y representación. 
El canal politemático Disney Cinemagic hace especial hincapié en las producciones cinematográficas para la confección de su parrilla de programación; una programación familiar dónde el 52’63\% de los productos analizados son aptos para un público de 3 a 6 años y el 47’36\% de los contenidos podríamos encasillarlos en un target de 7 a 12 años.

Disney XD es un canal más enfocado al segmento de edad que va de los 7 años en adelante (96'42\%) y donde el $100 \%$ de sus contenidos no están adaptados al Currículum Escolar de Primaria. De ahí que los contenidos estén más enfocados hacia la diversión y el entretenimiento de los niños:

"Incluye una irresistible combinación de series de acción real y de animación (...). Cuyos temas hacen referencia a sus metas, descubrimientos y logros, así como a los deportes, la aventura y el humor" (Inés Ramos, Directora de programación de los canales temáticos Disney).

En definitiva, podemos concluir este apartado destacando la buena distribución de contenidos atendiendo a distintos target potenciales de consumo televisivo, tratando de cubrir las expectativas y demandas del público infantil a través de sus distintos canales infantiles politemáticos. En este sentido, vemos cómo el salto de Disney Channel a la plataforma de TDT ha hecho que sus contenidos sean cada vez más generalistas, con el fin de abarcar un mayor segmento de audiencia pero sin llegar a perder la identidad que lo ha caracterizado durante años, de ahí que su parrilla esté compuesta por algunos de los productos audiovisuales que conforman el resto de los canales de su familia que no son de libre acceso sino que forman parte de las distintas plataformas de pago que operan en España.

Figura 2. Canales Disney

VARIABLE CURRÍCULUM ESCOLAR (EN \%)

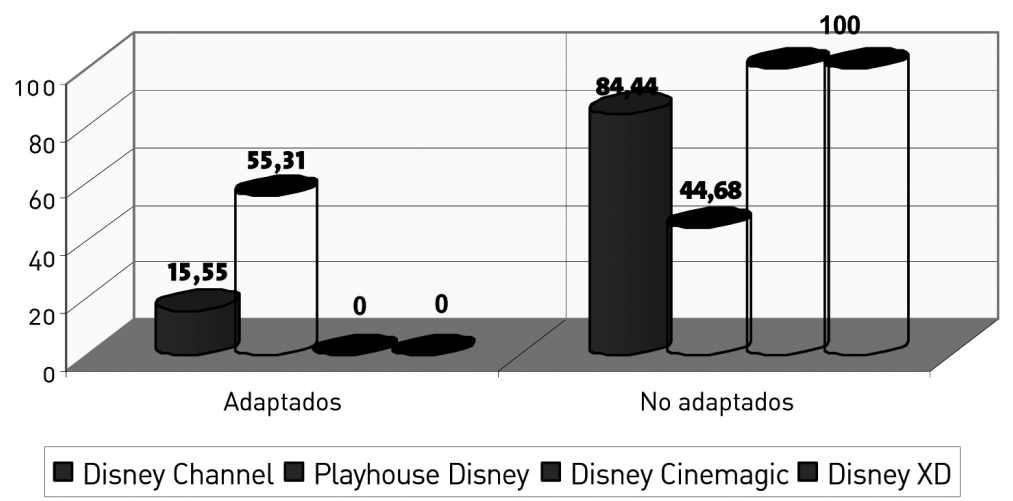




\section{Viacom Inc.: Nickelodeon y Nick JR}

El canal politemático Nickelodeon está caracterizado por dirigirse a un target mayor de 6 años. Encontramos cómo el 91'30\% de sus contenidos atienden a un público de entre 7 a 12 años y tan solo el 8'69 van destinados a niños de 3 a 6 años de edad. De ahí que Viacom disponga de otro canal exclusivo para niños preescolares de 3 a 6 años (Nick Jr.) que no son atendidos por Nickelodeon. En este sentido, Nickelodeon opta más por contenidos no basados en el Currículum Escolar con 95’65\% mientras que Nick Jr. hace una apuesta fuerte por incluir productos basados en el Currículum Escolar de infantil, por lo que dispone de un $71^{\prime} 42 \%$ de adaptados frente al $28^{\prime} 57 \%$ no adaptados.

Figura 3. Canales Viacom Inc.

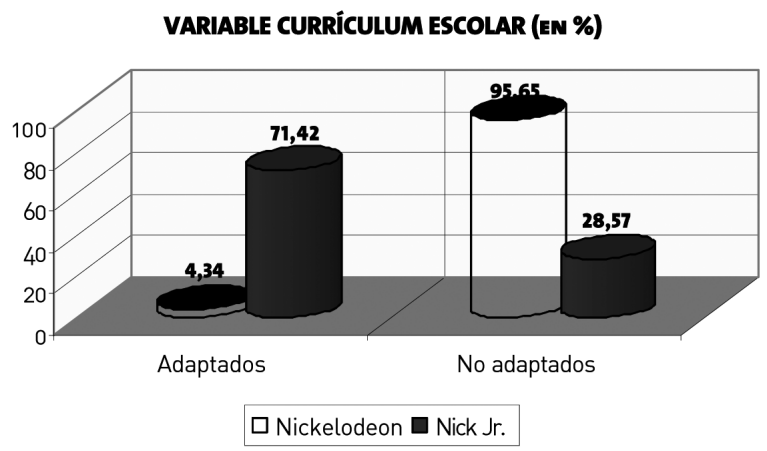

De este modo, tras el análisis de los contenidos de ambos canales, las áreas de conocimiento abordadas por los mismos quedan reflejadas en el correspondiente gráfico que nos muestra la clara tendencia a la inclusión en los productos audiovisuales de contenidos relacionados con el Conocimiento del Entorno, sobre todo cuando se hace referencia a niños en edad preescolar. 
Figura 4. Canales Viacom Inc.

\section{VARIABLE ÁREAS DE CONOCIMIENTO (EN \%)}

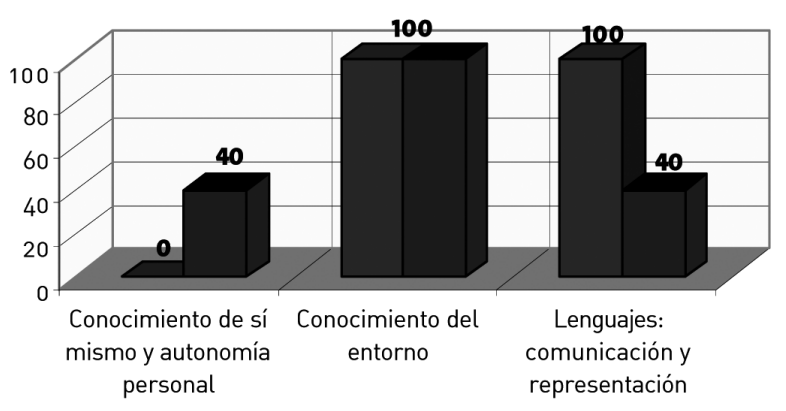

Nickelodeon $\square$ Nick Jr.

\section{Time Warner Company: Cartoon Network y Boomerang}

Los canales pertenecientes a este emporio se caracterizan por dirigirse a dos segmentos de público muy diferentes. El primero de ellos, Cartoon Network hace especial hincapié con sus contenidos a un segmento de edad de 6 años en adelante (95'23\%) al ser un canal pensado en exclusiva para niños más que para niñas, ya que su programación atiende a productos audiovisuales no adaptados al Currículum Escolar del niño, sino que se opta por la diversión y el humor.

En el segundo de los casos, Boomerang está destinado a un target de menor edad (de entre 3 a 6 años aproximadamente) y sus contenidos nos hacen vincularlo más hacia un público más femenino y familiar (90,9\%). De ahí que encontremos dos propuestas bien diferentes de canales infantiles politemáticos que tratan de cubrir las demandas de los más pequeños pero que en ninguno de los casos atienden a contenidos puramente educativos:

La audiencia de niños, históricamente, siempre se ha considerado de 4 a 12 años, pues a mediados de los 90 cuando hablabas de un canal para niños, tu target era de 4 a 12 años; lo que pasa es que los niños han ido evolucionando, han ido cambiando y eso es lo que ha provocado también la emergencia de canales temáticos que se han ido especializando (...). En el mundo de los niños también se ha ido segmentando y en función también de esa evolución, nosotros hemos ido construyendo dos propuestas diferentes (Vincent Sourdeau, Director de canales temáticos Cartoon Network y Boomerang). 


\section{Kidsco}

Este canal politemático atiende a un segmento de público potencial que va desde los 3 hasta los 12 años de edad. Así el 48'14\% de su programación va destinada al segmento de edad de los 3 a los 6 años, mientras el 51 '85\% de sus contenidos son aptos para un público mayor de 7 años. Dentro de esos intervalos de edad destacan los productos no adaptados al Currículum Escolar del menor en el 100\% de los casos analizados.

\section{BabyTV}

El público potencial de BabyTV suele ser menor de 2 años, al menos así lo corroboran los contenidos empleados para la confección de su parrilla de programación, ya que las 48 series analizadas son aptas para este tipo de público que viene siendo poco o nada estudiado en materia de televisión infantil. De este modo, constatamos cómo el 41’66\% de sus contenidos están adaptados al Currículum Escolar de la primera infancia y contribuyen al fomento del Conocimiento del entorno en un 95\% de los casos, así como un 60\% se corresponden con Lenguajes: comunicación y representación; es necesario aclarar que la manifestación de otros idiomas como el inglés dentro de los contenidos de BabyTV es más que evidente, puesto que se trata de un canal que bien podemos concebir como elemento para fomentar el aprendizaje de una segunda lengua (la gran mayoría de sus productos no están doblados al español), lo que supone una buena forma para que los pequeños españoles “hagan oído” y tomen un primer contacto con otro idioma.

\section{Conclusiones}

Tras el consiguiente acercamiento analítico al mundo de los canales infantiles politemáticos de televisión digital, que ha constituido nuestro universo a estudio, a continuación se exponen las conclusiones más relevantes que se pueden extraer del mismo:

Encontramos ofertas diferentes de contenidos conforme al estilo de cada uno de los canales infantiles politemáticos que han ocupado este estudio y en base al target potencial al que se dirigen. No obstante, se observan rasgos comunes en cuanto a las formas y tratamiento en los contenidos, así como en la estructura de los diversos canales infantiles; pese a ello hemos podido elaborar distintas clasificaciones atendiendo al género y al formato, a la publicidad y las autopromociones, y al segmento de edad y al Currículum Escolar que muestran la clara tendencia de estos modelos televisivos, lo que nos permite agrupar a los 11 canales de televisión digital estudiados en:

Infantil Politemático Educativo: Playhouse Disney, Nick Jr. y BabyTV. 
- $\quad$ Infantil Politemático de Entretenimiento: Boomerang.

- $\quad$ Infantil y Juvenil Politemático de Entretenimiento: CLAN TVE, Disney Channel, Kidsco.

- Juvenil Politemático de Entretenimiento: Cartoon Network, Nickelodeon, Disney XD.

Familiar Politemático de Ficción: Disney Cinemagic.

El segmento de edad empleado para confeccionar el canal politemático suele ser demasiado amplio, sobre todo cuando se hace referencia a los canales disponibles tanto en las plataformas en abierto (TDT), como en las de pago (ONO). Por lo que consideramos que se pierde la finalidad del canal politemático al interesarse por un amplio margen de público, ya que en ellos lo mismo te puedes encontrar un contenido óptimo para niños en edad preescolar que uno destinado a niños preadolescentes que sobrepasan los límites de las temáticas puramente infantiles. Por lo tanto, la denominación global que hoy día se hace de canal "infantil" estaría mal utilizada en este tipo de canales. A pesar de ello, reconocemos la buena utilización que se viene haciendo de la segmentación de edades, pues la mayoría de canales analizados fragmentan de forma óptima su audiencia.

Se constata una alarmante falta de variedad tanto de géneros como de formatos; la innovación escasea y se recurre de forma reiterada siempre a los mismos géneros como son la ficción y el entretenimiento, y a los mismos formatos: dibujos animados y series de ficción que predominan en las parrillas de programación de cada uno de los canales politemáticos estudiados. Sin embargo, encontramos cómo los contenidos que atienden al género educativo se hacen presentes sobre todo en los canales infantiles politemáticos destinados a un público en edad preescolar (de 3 a 6 años) y se constata su ausencia clara en aquellos enfocados a niños de 7 años en adelante donde la diversión y el humor son los parámetros que imperan a la hora de programar. Así mismo, el predominio del formato dibujos animados corrobora y constata lo ya puesto de manifiesto en diversidad de estudios sobre la percepción y la infancia que aseguran que los niños más pequeños gustan de la técnica del dibujo animado en las producciones audiovisuales.

Puesto que la publicidad forma parte de los espacios de continuidad televisiva, las inserciones publicitarias siguen predominando en las parrillas de programación de los canales infantiles politemáticos; pese a tratarse en su mayoría de canales de pago, éstos tienden a conseguir la máxima rentabilidad a través de los abonos y la inclusión de publicidad. No obstante, se rompe la lógica publicitaria a la que nos tenían acostumbrados los canales generalistas para buscar la máxima satisfacción del cliente/telespectador, lo que nos muestra una mejor distribución de los espacios publicitarios reduciendo su presencia en los canales para niños. Sin embargo, encontramos cómo los spots publicitarios de productos externos al canal se hacen más 
presentes en aquellos canales destinados a un segmento de audiencia de mayor edad (de 7 a 12 años) lo que va íntimamente relacionado con la adquisición de una mayor autonomía y un mayor poder de consumo por parte de los niños a partir de estas edades.

\section{Referencias bibliográficas}

Alcolea, G. (2003): La televisión digital en España. Sevilla: Comunicación Social.

Barroso, J. (1996): Realización de los géneros televisivos. Madrid: Síntesis.

Bartolomé, A. y Crescenzi, L. (2009): La televisión infantil frente los niños menores de 6 años. Informe LMI. Universidad de Barcelona. Disponible en: http://www.cac.cat/pfw_files/cma/recerca/estudis_recerca/Television_infantil_y_menores_de_6_a_os.pdf. Consultado el: 10/05/2010.

Blanco, I.; Römer, M. (2010): Los niños frente a las pantallas. Madrid: Universitas.

Boletín Oficial de la Región de Murcia Decreto no 286/2007 de 7 de septiembre de 2007. Currículum de Educación Primaria. Disponible en: www.carm.es/borm/documento?obj=anu\&id=324650. Consultado el: 10/05/2010.

Boletín Oficial del Estado, ORDEN ECI/3960/2007, de 19 de Diciembre del Ministerio de Educación y Ciencia. Currículum de Educación Infantil. Disponible en: http://www.boe.es/boe/dias/2008/01/05/pdfs/A01016-01036.pdf. Consultado el: $10 / 05 / 2010$.

Bustamante, E. y Álvarez, J.M. (1999): Presente y futuro de la televisión digital. Madrid: Edipo.

Cebrián, M. (2003): “Contenidos infantiles en televisión. Nueva técnica analítica global”, ZER Revista de estudios de comunicación. Disponible en: http://www.ehu.es/zer/zer15/articulo_3.htm. Consultado el: 10/05/2010.

Cebrián, M. (2004): Modelos de televisión: generalista, temática y convergente con Internet. Barcelona: Paidós.

CONECT (2008): "Estudio experimental sobre hábitos televisivos y eficacia publicitaria del target infantil: televisión de pago: un entorno de comunicación favorable y eficaz". Madrid. Disponible en: http://www.conect.es/2007/presentaciones/VEO\%20VEO\%20Presentaci\%F3n\%20(Maite\%20Rodr\%EDguez).pdf. Consultado el: 15/05/2010.

CONECT. (2010): “La televisión temática de pago en España 2009. Conectados a la eficacia”. FACTBOOK 2010. Disponible en: http://www.conect.es/pdf/factbook_10.pdf Consultado el: 15/05/2010.

De Andrés, T. (2006): El desarrollo de la inteligencia fílmica. La comprensión audiovisual y su evolución en la infancia y adolescencia. Madrid: CNICE.

Francés, M. (Coord.). (2009): Hacia un nuevo modelo televisivo. Contenidos para la Televisión Digital. Barcelona: Gedisa.

Fuente, C. (Coord.). (2009): Infancia y televisión. Políticas de protección de los menores ante los contenidos audiovisuales. Madrid: Fragua. 
Galindo Rubio, F. (2004): Comunicación audiovisual corporativa. Cómo audiovisualizar la identidad de las organizaciones. Salamanca: Publicaciones Universidad Pontificia de Salamanca.

García, F. y De Andrés, T. (2000): La representación del niño en los medios de comunicación. Madrid: Huerga \& Fierro.

García, MC. (2000): Televisión, violencia e infancia. El impacto de los medios. Barcelona: Gedisa.

Gómez, M. (2005): “Los nuevos géneros de la neotelevisión”, Área Abierta, n. 12. Disponible en: http://revistas.ucm.es/ inf/15788393/articulos/ARAB0606130002A.PDF Consultado el: 15/05/2010.

González, C. (2005): "Programación infantil: entre la educación y el entretenimiento, Comunicar: Revista científica Iberoamericana de comunicación y Educación, n. 25, 2. Huelva. Disponible en: http://redalyc.uaemex.mx/redalyc/pdf/ 158/15825120.pdf. Consultado el: 15/05/2010.

González, C. y Fanjul, C. (2009): "Marcas televisivas. La gestión de la continuidad en el medio televisivo español. Revista Latina de Comunicación Social, n. 64, pp. 894-908. La Laguna. Tenerife. Disponible en: http://www.revistalatinacs.org/ 09/art/869_UJI/70_95_Gonzalez_y_Fanjul.html. Consultado el: 22/05/2010.

Impulsa TDT. (2010): Anuario TDT 2010. TDT Informe Final 2010. Madrid.

Jiménez, E. (2005): "La televisión ante un cambio de escenario. Análisis en perspectiva: de cómo la llegada de las privadas modificó los hábitos de producción y consumo televisivo”. Universidad de Navarra. Disponible en: www.unav.es/fcom/ cicom/.../estefanía\%20jiménez.pdf Consultado el: 10/05/2010.

Katz, D.; Busemann, A.; Piaget, J. e Inhelder, B. (1985), [1960]: Psicología de las edades. Del nacer al morir. Madrid: Morata.

López, L. y González, C. (2005): “El posicionamiento en 20: las autopromociones de las cadenas de televisión”, Comunicar, n. 25, Huelva. Disponible en: http://redalyc.uaemex.mx/pdf/158/15825080.pdf. Consultado el: 22/05/2010.

Marín, V. (2005): “Las series animadas de televisión y su valor educativo", Comunicar. Huelva. Disponible en: http://redalyc.uaemex.mx/pdf/158/15825190.pdf Consultado el: 10/05/2010.

Marta, C. (2005): Análisis de la audiencia infantil: de receptores de la televisión a perceptores participantes. Disponible en: http://eprints.ucm.es/tesis/inf/ucm-t28490.pdf. Consultado el: 10/05/2010.

Melgarejo, I. (2010): Canal Infantil Politemático de Televisión Digital. Clasificación y aproximación a una definición. Trabajo de Suficiencia Investigadora, Directora: Rodríguez, MM. Facultad de Ciencias Sociales y de la Comunicación, Universidad Católica San Antonio de Murcia.

Melgarejo, I. y Rodríguez, MM. (2010): "La calidad como consecuencia de una buena segmentación de edades en la televisión temática infantil: Playhouse Disney y los contenidos preescolares”. II Congreso Internacional AE-IC Málaga 2010. Facultad de Comunicación de la Universidad de Málaga. Disponible en: http://www.aeic2010malaga.org/upload/ ok/177.pdf. Consultado el: 10/03/2010.

Merayo, A. (Ed.). (2007): El gato en el microondas. Enseña a tu hijo a convivir con los medios. Barcelona: Nabla. 
Mora-Figueroa, B. (2009): El mercado global de la comunicación. Éxitos y fracasos. Navarra: Eunsa.

Núñez, L. y Torrecillas, T. (2007): “El contexto de recepción infantil”, Telos: Cuadernos de comunicación e innovación, n. 73. Disponible en: http://sociedadinformacion.fundacion.telefonica.com/telos/articulocuaderno.asp@idarticulo=1\&rev= 73.htm. Consultado el: 8/04/2010.

Palacio, M. (2008): “¿Qué es la televisión temática?”. I Foro de la Televisión Temática en España. San Lorenzo del Escorial. Madrid. Disponible en:http://www.conect.es/2007/presentaciones/Qu\%E9\%20es\%20la\%20televisi\%F3n\%20tem\%E1tica \%20(Profesor\%20Palacio).pdf. Consultado el: 22/05/2010.

Pérez, JR. y Núñez, L. (2006): “Lo que los niños ven en la televisión”, Zer: Revista de estudios de comunicación, n. 20. Disponible en: http://www.ehu.es/zer/zer20/zer20_11_ladeveze.pdf. Consultado el: 08/04/2010.

Piaget, J. (2001), [1969]: Psicología y pedagogía. Barcelona: Biblioteca de bolsillo.

Rodríguez, MM. y Correyero, B. (Coord.). (2008): Medios Responsables: panorama Internacional de contenidos programáticos para menores, Sphera Publica, n. 8. UCAM.

Rodríguez, MM. y Melgarejo, I. (2010): “Cine infantil: aproximación a una definición”, Doxa Comunicación, Revista interdisciplinar de estudios de comunicación y ciencias sociales. CEU, n. 10. pp. 167-181.

Sánchez, JL. (2002): Historia del Cine. Teoría y géneros cinematográficos, fotografía y televisión. Madrid. Alianza.

Sánchez-Tabernero, A. (2008): Los contenidos de los medios de comunicación. Calidad, rentabilidad y competencia. Barcelona: Deusto.

Sánchez-Tabernero, A. (2009): "La televisión en España. Informe (2009)". Universidad de Navarra. Disponible en: http://www.iese.edu/en/files/01_UTECA_ALFONSO_SANCHEZ_TABERNERO_tcm4-40108.pdf. Consultado el: $10 / 05 / 2010$.

Tur, V. (2004): Comunicación publicitaria de juguetes en televisión. Universidad de Alicante: Alicante.

Tur, V. y Ramos Soler, I. (2008): Marketing y niños. Madrid: ESIC.

Ventura, R. (2001): La televisión por cable en España. Barcelona: EUB.

Walzer, A. (2008): “Televisión y menores: Análisis de flujos de programación y de recepción. Estudio comparado: 20032007", ZER Revista de estudios de comunicación, n. 24. Disponible en: http://www.ehu.es/zer/zer24/zer24-walzer.htm. Consultado el: 10/05/2010. 